\title{
Vol. 69, No. SS-4
}

In the Surveillance Summary "Prevalence of Autism Spectrum Disorder Among Children Aged 8 Years — Autism and Developmental Disabilities Monitoring Network, 11 Sites, United States, 2016," errors occurred in the Abstract and Table 2 and Table 3.

On page 1 in Results, the percentage of girls with intellectual disability should have been $\mathbf{3 9 \%}$. On page 11 , the second footnote of Table 2 should have referenced Supplementary Table 7. On page 11, the fourth column header in Table 3 should have been IQ $\leq 70$. 